\title{
Comparison of Detection and Classification of Hard Exudates using Artificial Neural System vs SVM Radial Basis Function in Diabetic Retinopathy
}

\author{
V.Sudha ${ }^{*}$, T. R.Ganesh Babu², R. Raja ${ }^{3}$ \\ ${ }^{I^{*}}$ Department of Electronics \& Communication Engineering, Pavai College of Technology, Namakkal (Dt), INDIA. \\ ${ }^{2}$ Department of Electronics \& Communication Engineering, Muthayammal Engineering College, Namakkal (Dt), INDIA. \\ ${ }^{3}$ Department of Electrical and Electronics Engineering, Muthayammal Engineering College, Namakkal (Dt), INDIA.
}

\begin{abstract}
Diabetic Retinopathy (DR) is a disease that occurs in the eye which results in blindness as it passes to proliferative stage. Diabetes can significantly result in symptoms like blurring of vision, kidney failure, nervous damage. Hence it has become necessary to identify retinal damage that occurs in diabetic eye due to raised glucose level in its initial stage itself. Hence automated detection of anamoly has become very essential. The appearance of crimson and yellow lesions is considered as the earliest symptoms of DR are called as hemorrhages and exudates. If DR is analysed at initial stage, blindness does not occur. The damage in retina can hinder the light that passes through nerves of the eye leading to visual loss. The motivation behind this research is to reduce the number of false positives by accurate detection which is possible using proposed fuzzy system based on ANN. Though several classifiers are available to detect the exudates this paper makes analysis of support vector machine using radial basis kernel function with proposed ANN technique. Also, adaptive neuro fuzzy inference system segmentation is performed after feature extraction technique, which makes classifer to outperform. The evaluation results showed that proposed artificial neural network based on fuzzy approach attained significant results compared to other classifiers. Moreover, the proposed algorithm has significant accuracy of $94 \%$ and minimum error rate has been observed.
\end{abstract}

Keywords: Support vector machine, radial basis function, fuzzy inference systems, neural networks, exudates, segmentation.

\section{Introduction}

Diabetes is a common disease that affects quite large population across the world. Raised glucose levels always lead to presence of anamolies in retinal layer of eye. Initial stage of retinopathy is characterized by hard, soft exudates, microaneurysms, haemorrhages. When there occurs growth of tiny blood vessels, it is microaneurysms. Also, leakage of blood from retinal blood vessels is haemorrhage. There are different stages in retinopathy. In stage of nonproliferative retinopathy [1], retinal vessels are damaged hence fatty and protein-based particles are leaked called as exudates. If accumulation of exudates occurs in centre of the retina, it can result in loss of vision in the nonproliferative stage of DR. Exudates are characterised with vascular damage patches resulting in leakage which is viewed as random yellow patches of various sizes and shapes. Exudates are related to retinal edema and visual loss, which is considered as most

"Corresponding Author: e-mail: vsudhaece@gmail.com ISSN 2320-7590

(C) 2020 Darshan Institute of Engg. \& Tech., All rights reserved important retinal lesion detection. The Diabetic Macular Edema is based on feature vector classification produced for images. [2] This exudates are segmented using feature based technique. This comprises of morphological method that uses algorithm based on SVM.[3].

Here, two classifier techniques are compared with Bayesian and Artificial neural network and performance was analysed. [4]. This methodology used neural network for classification of diseases to get better result than Bayesian classifier. To identify the feaures present in image several preprocessing techniques are used. Using fuzzy, this segmentation process is done.[5] To identify the retinal bright lesions a minimumdistance discriminant classifier was used. The drusen dependent on 16 matrix type was used and trained using neural networks. [6] It achieved $91 \%$ accuracy for patch resolution performance using small number of upto 15 retinal images. An automated machine learning-based system is capable of detecting both the types of exudates and distinguish from drusen that is present in color images. 
A diabetic patient has developed approaches to improve the retinal experts performance level. Using additional data sets, machine learning technique can be improved for identifying clinically significant lesions that are bright, thereby improving diagnosis at an early stage to avoid visual loss that occur in diabetes patients. Figure1 illustrated normal and abnormal image.

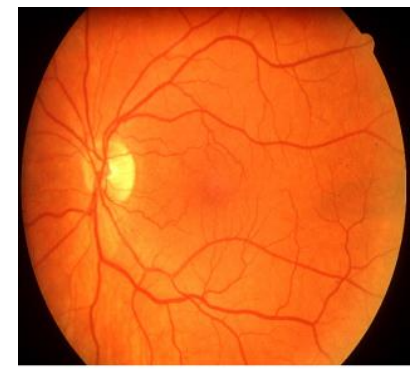

(a)

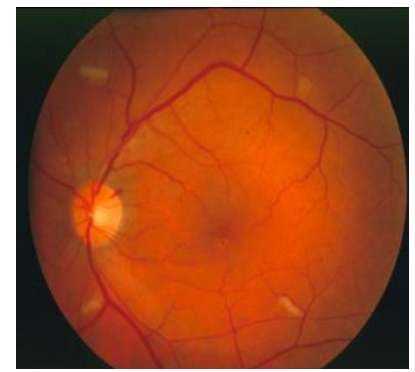

(b)
Figure 1: Retinal Images a) Normal b) Image showing abnormality

Wang et al. [9] to obtain graded features and classify using random forest that is trained using convolutional neural networks. To extract the features it used 6 convnets layers along with a subsequent sub-sampling layer. DRIVE and STARE databases were used to acquire accuracy of $98.1 \%$ and $97.2 \%$ respectively. Complexity is increased by additional preprocessing levels through technique of deblurring prior to detection, segmentation of microvascular condition, improving light intensity using mathematical model and morphological reconstruction. To detect darkcolored lesions named as microaneurysms and haemorrhages thresholding technique along with, adaptive preprocessing was used $[10,11]$ after that the vessels were removed set of input Structures. Using multilayer perceptron analysis vessels are identified [10] along with multiscale morphological closing operation [11]. The major drawback being that most of the false positives detected during segmentation of vessels are identified as lesions. Due to this, lesions are lost and are not retrieved in subsequent processing of blood vessels.

Table I Details of Image Dataset Collections

\begin{tabular}{|c|c|c|c|c|c|}
\hline \multirow{2}{*}{ Database } & \multirow{2}{*}{$\begin{array}{c}\text { Normal } \\
\text { Images }\end{array}$} & \multicolumn{4}{|c|}{ Abnormal Images } \\
\cline { 3 - 6 } & & Exudates & \multicolumn{2}{c|}{$\begin{array}{c}\text { otton Wool } \\
\text { Spot }\end{array}$} \\
\cline { 3 - 6 } & & Hard & Soft & Few & $\begin{array}{c}\text { Man } \\
\mathbf{y}\end{array}$ \\
\hline STARE & 110 & 40 & 47 & 10 & 40 \\
\hline MESSIDOR & 83 & \multicolumn{2}{|c|}{83} & \multicolumn{2}{|c|}{75} \\
\hline \multirow{2}{*}{ Total } & 200 & 170 & \multicolumn{2}{|c|}{125} \\
\cline { 2 - 6 } & Normal =193 & \multicolumn{3}{|c|}{ Abnormal $=295$} \\
\hline
\end{tabular}

\section{Image Preprocessing}

The data set required for analysis is obtained from publically available database such as MESSIDOR with largest resolution of $2204 \times 1536$ and STARE of $600 \times 700$ resolution database. Preprocessing is a technique to enhance picture information for processing the image. Picture preprocessing techniques can be characterized into four classes: pixel, geometric variation. The original RGB fundus image to HSI image space used colour space transition. The noise band containing noise is removed using median filter over the full image. To enhance the contrast, Contrast limited Adaptive Histogram equalization technique is performed. This technique is different from Gaussian function based methods, as CLAHE avoids saturation in similar areas of the image. As optic disc and exudate uses same characteristics of intensity, RGB is converted to HSI image prior to processing. This conversion does involve RGB to grayscale conversion. Binary operation performed in the HSI image can reduce the effect of noise and artifacts in image. Optic disc can be removed by using large circular components present in the fundus image. Exudates are classified as soft and hard exudates finally using SVM classifier.

\section{Classification using SVM Radial basis function}

Classification algorithms are used to get images required of various sizes. Classifier of supervisor type needs user input. Unsupervised classifier needs large set of information to estimate the structure. SVM is predicted as a supervised learning technique that is used for regression and classification. A support vector machine improves the detection accuracy by using a regression and classification technique utilising machine learning technique. The technique is simple, with no optimum value with good error controllability. SVM requires kernel function to reduce its drawbacks. Kernel function of SVM is Radial basis function is the kernel function that used Cross validation technique.

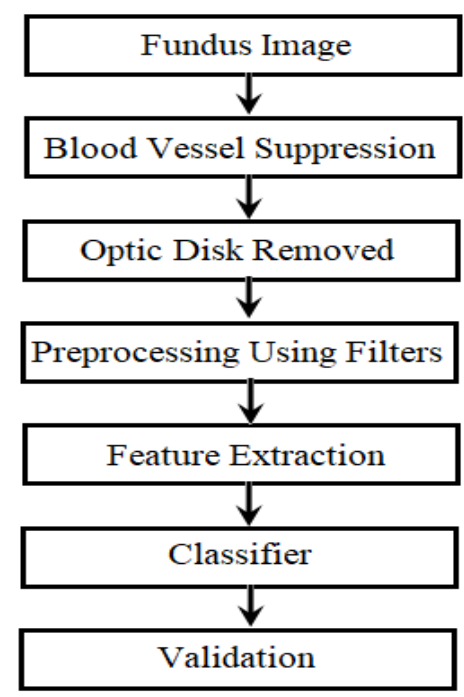

Figure 2 : Flow diagram of proposed method 


\section{Feature Extraction for ANN}

Following the collected dataset and preprocessing certain features respective of shape,intensity, gray tone, and parameters of fourier transform are extracted.

Table II Feature description

\begin{tabular}{|c|c|c|}
\hline \multicolumn{3}{|c|}{ Table II Feature description } \\
\hline Symbol & Feature & Description \\
\hline \multirow{3}{*}{$\begin{array}{l}\text { Parameters } \\
\text { for shape }\end{array}$} & $\mathrm{F}_{1}$ - Area & Sum of lesion pixels \\
\hline & $\mathrm{F}_{2}$ - Eccentricity & $\begin{array}{c}\text { Distance between foci / } \\
\text { major axis }\end{array}$ \\
\hline & $\mathrm{F}_{3}-$ Diameter & Lesion Diameter \\
\hline \multirow{2}{*}{$\begin{array}{l}\text { Intensity } \\
\text { color }\end{array}$} & $\begin{array}{l}\mathrm{F}_{4}-\text { Mean } \\
\text { intensity }\end{array}$ & \multirow{2}{*}{$\begin{array}{l}\text { color and intensity } \\
\text { properties }\end{array}$} \\
\hline & $\mathrm{F}_{5}$ - Energy & \\
\hline \multirow{2}{*}{$\begin{array}{l}\text { Gray tone } \\
\text { texture }\end{array}$} & $\mathrm{F}_{6^{-}}$Coarseness & \multirow{2}{*}{$\begin{array}{l}\text { visible image texture } \\
\text { properties }\end{array}$} \\
\hline & $\mathrm{F}_{7}$ - Complexity & \\
\hline \multirow{2}{*}{$\begin{array}{c}\text { Fourier } \\
\text { parameters }\end{array}$} & $\mathrm{F}_{8}$ - Radial sum & \multirow{2}{*}{$\begin{array}{l}\text { image texture property } \\
\text { in Fourier domain }\end{array}$} \\
\hline & $\begin{array}{l}\text { F9- Angular }_{\text {sum }} \\
\text { sul }\end{array}$ & \\
\hline
\end{tabular}

\section{Classification using Artificial neural networks}

The performance of artificial neural classifier was analysed based on IF-THEN condition. Two factors such as basis and resultant factors are used. The basis factor is based on Fuzzy rule based on IF condition which is given as input and another Fuzzy condition is based on THEN condition is thereby given as resultant factor. The foremost significance of the classifier is that it sufficiently increases capacity of dataset that is used for training and by changing the basis functions and Resultant factors the classifier can operate. Here MESSIDOR database is used for analysis that is publicly available. The adaptive type of fuzzy classifier network has total layers of five in number to perform many computational functions using input signals. The layer three can calculate the Fuzzy strength based on certain conditions that is based on the updations of second layer.

The layer four computes the subsequent factors based on THEN condition of the Fuzzy condition logic rules. The layer five computes the aggregate results of previous nodes as one value, which finds the input feature vector belonging to a class. The diagnostic results of classifier predicted included normal or abnormal image with exudates in Diabetic retinopathy. The first layer represents the basis factor and the output result is extracted from the output layer which is represented by subsequent factors corresponding to
THEN condition. The classifier accuracy is dependent on proper selection of highly unique feature vector and via thorough training process.

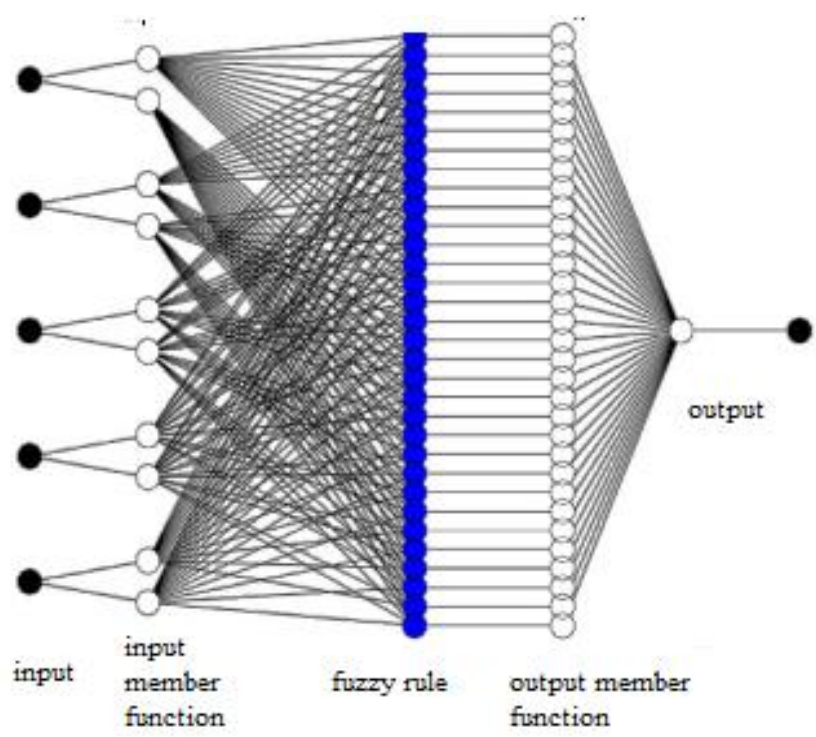

Figure 3. Structure of ANFIS system

Figure 3 shows ANFIS system that operates on 32 AND fuzzy rules which uses membership functions to get output, that has 5 inputs.

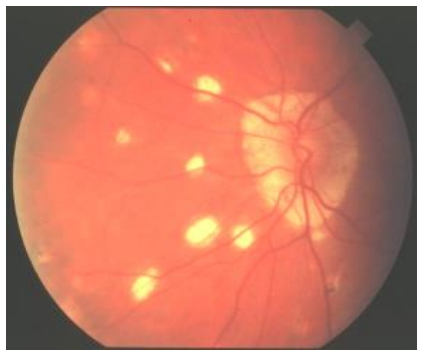

(a)

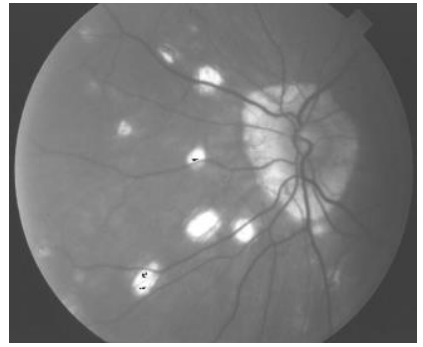

(b)
Figure 4: a) RGB fundus image b) Green channel

Figure 4 showed green channel extraction from RGB fundus. Here green channel is extracted as it possess better contrast level and good visibility of fundus area.

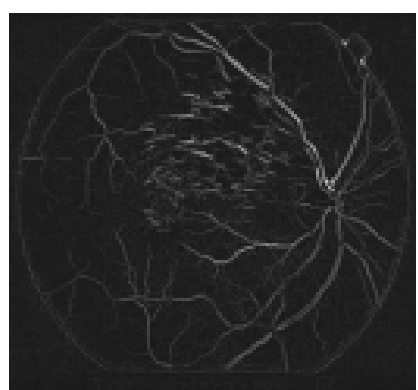

(a)

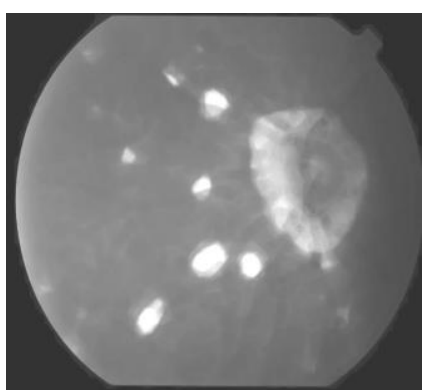

(b)
Figure 5: a) blood vasculature b) Non blood vessels 
Figure 5 showed blood vasculature and non-blood vessels. Using morphological filter blood vessels are extracted so as to identify the exudates more efficiently. Using contourlet type of transform the morphological segmentation is performed.

Following the classification, validation is performed to identify the results of diagnosis into two classes where first includes Healthy eyes and second includes exudates lesion.

Table I: Confusion Matrix

\begin{tabular}{|c|c|c|c|}
\hline \multirow{4}{*}{ Samples } & \multicolumn{3}{|c|}{ Predictions of classifiers } \\
\cline { 2 - 4 } & $\begin{array}{c}\text { Abnormal } \\
\text { Ones }\end{array}$ & $\begin{array}{c}\text { Abnormal } \\
\text { Images }\end{array}$ & $\begin{array}{c}\text { Normal } \\
\text { Images }\end{array}$ \\
\cline { 2 - 4 } & $\begin{array}{c}\text { TN } \\
\text { (True negative) }\end{array}$ & $\begin{array}{c}\text { FP } \\
\text { (False Positive) }\end{array}$ \\
\cline { 2 - 4 } & $\begin{array}{c}\text { Normal } \\
\text { Ones }\end{array}$ & $\begin{array}{c}\text { FN } \\
\text { (False negative) }\end{array}$ & $\begin{array}{c}\text { TP } \\
\text { (True positives) }\end{array}$ \\
\hline
\end{tabular}

Four parameters are to taken into account to compute the accuracy. The confusion matrix in Table- 1 showed the samples for prediction of classes as True Negative, False Positive, False Negative and True Positive. TN depicts the number of correct rejections made in the decision during diagnosis. FP indicates the number of false alarms made during the decision of diagnosis. FN represents the misses that is made wrong during the time of diagnostic decision. TP indicates the count correctly identified as normal images which is hits along diagnosis direction.

Table II: Comparison of Classifier Algorithms

\begin{tabular}{|c|c|c|c|}
\hline Parameters & ANFIS & $\begin{array}{c}\text { RBF Kernel } \\
\text { SVM }\end{array}$ & $\begin{array}{c}\text { Naive } \\
\text { Bayes }\end{array}$ \\
\hline $\begin{array}{c}\text { Classification } \\
\text { Error }\end{array}$ & 0.07 & 0.25 & 0.36 \\
\hline Accuracy & 0.94 & 0.75 & 0.72 \\
\hline Specificity & 0.92 & 0.906 & 0.88 \\
\hline Sensitivity & 0.92 & 0.62 & 0.60 \\
\hline
\end{tabular}

Table II shows the comparison of classifier algorithms for various performance parameters with Naïve bayes classifier method. The results prove that artificial neural network performance is better compared with other classifiers.

\section{Conclusions}

The confusion matrix depicted above showed the various performance metrics like specificity, sensitivity and accuracy. We find that artificial inference system outperforms Radial basis function based on SVM kernel and Naïve Bayes method in terms of classification. The proposed classifier based on fuzzy inference system showed better results in terms of accuracy for correct prediction of patients with anamoly. Also we find specificity, sensitivity corresponding to patients without disease and with disease were estimated as $92 \%$ The results prove that fuzzy inference system based on neural networks can perform much better compared with conventionally available classifier techniques like SVM with radial basis function and naïve bayes technique. The analysis proved that less error rate was involved in proposed than conventional technique.

\section{References}

1. S. Lee, E. T. Lee, Y. Wang, and R. Klein, "Computer classification of nonproliferative diabetic retinopathy," Arch. Ophthalmol., vol. 123, no. 6, pp. 759-764, 2005.

2. Fabrice Meriaudeau, Thomas P. Karnowski, Yaqin Li, SeemaGarg, Kenneth W. Tobin Jr., Edward Chaum, Exudate-based diabetic macular edema detection in fundus images using publicly available datasets"e, Medical Image Analysis, Vol. 16,No. 1, pp. 216-226, 2012.

Atul Kumar, Abhishek Kumar, Manish Srivastava, A Segment based Technique for detecting Exudate from Retinal Fundus image"e, Procedia Technology, Vol. 6, pp. 1-9, 2012.

3. Jagadish Nayak \& P Subbanna Bhat \& Rajendra Acharya U \& C. M. Lim \& Manjunath Kagathi "Automated Identification of Diabetic Retinopathy Stages Using Digital Fundus Images", J Med Syst 2008, 107-115

4. Alireza Osare et al., "A Computational IntelligenceBased Approach for Detection of Exudatesin Diabetic Retinopathy Images, IEEE Transactions on Information Technology in Biomedicine, Vol. 13, No. 4, pp 535-545, July 2009.

5. M.Niemeijer, B. Ginneken,S.R. Russell, S.A.Maria and M.D.Abramoff, "Automated detection and differentiation of drusen, exudates, and cotton-wool spots in digital color fundus photographs for diabetic retinopathy diagnosis," Investigative Opthalmology \& Visual Science, vol. 48, no. 5, pp. 2260-7, 2007.

6. Yang.Y, Li. T, Li. W, Wu. H, Fan. W, Zhang. W, "Lesion detection and grading of diabetic retinopathy via two-stage deep convolutional neural networks" In Proceedings of the International Conference on Medical Image Computing and Computer Assisted Intervention, USA, 21-26, pp. 533-540, 2017.

7. Wang.S, Yin.Y, Cao.G, Wei.B, Zheng.Y, Yang.G "Hierarchical retinal blood vessel segmentation based feature and ensemble learning", 
Neurocomputing, vol. 226, no.22, pp. 270-272, 2017.

8. C.Sinthanayothin et al., "Automated detection of diabetic retinopathy on digital fundus images." Diabetic medicine: a journal of the British Diabetic Association, vol. 19, no. 2, pp. 105, 2002.

9. S.Ravishankar,A.Jain,andA.Mittal,“Automated feature extraction for early detection of diabetic retinopathy in fundus images," IEEE Conference on Computer Vision and Pattern Recognition, pp. 2107, 2009.

10. H. Wang, H. Hsu, K. Goh, and M. Lee, "An effective approach to detect lesions in retinal images,"in Proc. IEEE Conf. Comput. Vis. Pattern Recogn., Hilton Head Island, SC, 2000, vol. 2, pp. 181-187.

11. Joshi.S \& Karule P.T, A review on exudates detection methods for diabetic retinopathy. Biomedicine\& Pharmacotherapy, 97, 1454, 2018.

12. Sridevi,S.\& Nirmala.S. ANFIS based decision support system for prenatal detection of Truncus
Arteriosus congenital heart defect. Applied Soft Computing, 46, 577-587, 2016.

13. B.Verma and P. Zhang, "A novel neural-genetic algorithm to find the most significant combination of features in digital mammograms," Appl. Soft Comput., vol. 7, pp. 612-625, 2007

14. Asha PR, Karpagavalli S, "Diabetic retinal exudates detection using machine learning techniques", In Advanced Computing and Communication Systems, International Conference on. 2015,1-5.

15. M.Niemeijer,B.V.Ginneken,S.R.RusselM.Suttorp, and M.D.Abramoff, "Automated detection and differentiation of drusen, exudates and cotton-wool spots in digital color fun- dus photographs for diabetic retinopathy diagnosis," Invest. Ophthalmol. Vis. Sci., vol. 48, pp. 2260-2267, 2007.

\section{Biographical notes}
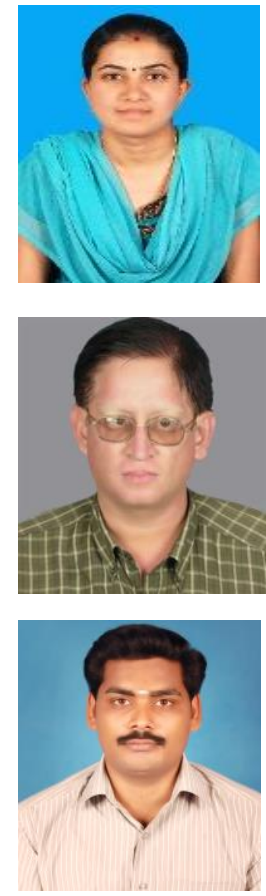

V.Sudha is currently pursuing her Ph.D under Anna University, Chennai in the Dpartment of Electronics \& Communication Engineering in Pavai College of Technology. She possess 9 years of teaching experience and has made significant contribuions in the area of Deep learning using Convolutional neural networks, Medical image analysis for Diabetic Retinopathy. She has made significant publications in International and National journals.

T.R. Ganesh Babu, Professor, Muthayammal Engineering College, India has completed his Ph.D. in Medical Electronics from Anna University, India on 2011. He is supervisor for scholars pursuing their Ph..D programmes. He possess about 20 years of teaching experience in engineering colleges. He has more than 35 journal publications in reputed journals. His area of interest includes Medical Image processing, Deep learning. He has been editor and reviewer for various journals and also has written many books.

R.Raja has received his Ph.D from Anna University Chennai in Faculty of Electrical Engineering in the year 2020. He is Assistant Professor in Electrical engineering department at Muthayammal Engineering College,Rasipuram India. His area of interest include Power electronics, Image processing, Artificial neural networks. He has made significant publications in International and National journals. 